\title{
Uso de un nuevo protocolo clínico fotográfico-computacional en el registro de la fluorescencia visible inducida por radiación ultravioleta en dientes humanos.
}

\author{
Use of a new clinical photographic-computational protocol to \\ register the ultraviolet-induced visible fluorescence in human \\ teeth.
}

\author{
Pablo Angel ${ }^{1 *}$, Javier Martín ${ }^{1}$, Natalia Lobos ${ }^{2}$, Roque Arias ${ }^{1}$, Erick Ampuero ${ }^{1}$, Cristian Salgado ${ }^{1}$
}

\author{
1. Departamento de Odontología Restauradora, \\ Facultad de Odontología, Universidad de Chile, \\ Santiago, Chile. \\ 2. Práctica privada, Iquique, Chile \\ * Correspondencia Autor: Pablo Angel | Dirección: \\ Olivos 943, Independencia, Santiago, Chile. I \\ Teléfono: +562 2978 1742, | E-mail: pangel@ \\ odontologia.uchile.cl. \\ Trabajo recibido: 30/08/2020 \\ Trabajo revisado: 04/10/2020 \\ Aprobado para su publicación el 08/11/2020
}

\begin{abstract}
RESUMEN
Los dientes humanos experimentan fluorescencia visible al ser sometidos a radiación ultravioleta, la cual varía de color a medida que el diente envejece. Objetivo: el presente estudio buscó desarrollar un protocolo clínico sencillo usando fotografía digital y análisis computacional para determinar la fluorescencia visible inducida por radiación ultravioleta (FVIUV) en dientes anteriores de pacientes de distintos grupos etarios. Materiales y Métodos: 35 participantes de 5 grupos etarios fueron reclutados. 70 incisivos centrales superiores fueron fotografiados utilizando como fuente de iluminación luz UV. Cada imagen obtenida fue analizada con el software computacional ImageJ para obtener las coordenadas de color en el espacio CIELab para la corona completa. Los datos obtenidos fueron analizados mediante las pruebas estadísticas de ANOVA y post-hoc de Tukey HSD. Resultados: Se obtuvieron las magnitudes de FVIUV en las tres dimensiones del espacio de color CIELab en la corona completa. Esta FVIUV se manifestó en el espectro del color verdeazulado para todos los grupos etarios, los cuales presentaron diferencias de saturación y luminosidad según tramo etario. Conclusiones: El protocolo aplicado en presente estudio permitió la cuantificación de la FVIUV dentaria en distintos grupos etarios.
\end{abstract}

PALABRAS CLAVE

Fluorescencia; Grupos etarios; Rayos Ultravioleta; Color, Diente; Estética.

Int. J. Inter. Dent Vol. 14(2); 148-152, 2021.

\begin{abstract}
Human teeth experience visible fluorescence when subjected to ultraviolet radiation, which varies in color as the tooth ages. Objective: The present study sought to develop a simple clinical protocol using digital photography and computational analysis to determine the difference in visible fluorescence induced by ultraviolet radiation (FVIUV) in anterior teeth of patients of different age groups. Materials and Methods: 35 participants from five age groups were recruited. 70 upper central incisors were photographed using UV light as the illumination source. Each image obtained was analyzed with Image J computer software to obtain the color coordinates in CIELab space for the entire crown. The data obtained was analyzed using the ANOVA and Tukey HSD post-hoc statistical tests. Results: Visible fluorescence magnitudes were obtained in the three dimensions of the CIELab color space in the complete crown. This FVIUV was registered in the spectrum of the blue-green color for all age groups, which presented differences in saturation and luminosity according to age group. Conclusions: The protocol applied in this study allowed the quantification of dental FVIUV in different age groups.

KEY WORDS
\end{abstract}

Fluorescence, Age Groups; Ultraviolet Rays; Color; Tooth; Esthetics.

Int. J. Inter. Dent Vol. 14(2); 148-152, 2021.

\section{INTRODUCCIÓN}

La fluorescencia es un fenómeno óptico en que un objeto refleja una radiación de longitud de onda mayor que la longitud de onda usada como iluminante ${ }^{(1-3)}$. Si el objeto es iluminado con radiación ultravioleta (UV longitud de onda 100 a 380 nm) y emite o refleja luz visible (longitud de onda 400 a $760 \mathrm{~nm}$ ) el fenómeno se denominará fluorescencia visible inducida por luz ultravioleta (FVIUV) $)^{(3)}$.

Cuando la estructura dentaria natural es estimulada por radiación ultravioleta, exhibe FVIUV en el rango de $350-400 \mathrm{~nm}^{(4,5)}$, reflejando luz blanco-azul del espectro electromagnético(6). Si bien es cierto que bajo la luz natural diurna esta FVIUV no genera grandes cambios en lo apariencia dentaria ${ }^{(7)}$, bajo ambientes con fuentes de iluminación artificial con mayor componente porcentual de radiación UV, como ampolletas fluorescentes, 
flashes de cámaras fotográficas o luces de estudio de alta intensidad ${ }^{(8-10)}$, los dientes se pueden apreciar más blancos o brillantes, contrastando en diversos grados con respecto a los materiales restauradores presentes ${ }^{(9,11)}$.

En el pasado la constatación clínica de este fenómeno resultaba impensable dado el gran tamaño de tubos o lámparas UV disponibles, sin embargo, el advenimiento de pequeñas linternas LED emisoras de radiación UV de adecuada intensidad y manejo para uso intraoral ha permitido el rápido examen revelando características que de otra forma resultaría imposible(11)

A pesar de lo anterior, persiste el problema de cómo cuantificar lo más objetiva y reproduciblemente este fenómeno. Al respecto, existen propuestas de utilizar un método combinado de fotografía digital y software computacional|(12)

Para medir la expresión visible de esta fluorescencia inducida por radiación UV, el espacio de color CIELab resulta el más adecuado. En éste, la coordenada $L$ nos informa de la luminosidad del objeto estudiado, siendo usada como medida de la fluorescencia y reflectancia ${ }^{(13)}$, y las coordenadas $a^{*} \mathrm{y} \mathrm{b}^{*}$ se refieren a la saturación y el tono de color.

En el presente estudio nos proponemos realizar in vivo un protocolo simple de registro y cuantificación de la FVIUV en el espacio de color CIELab, usando fotografía y un software de libre disposición.

Habiéndose reportado que los dientes experimentan cambios de fluorescencia visible al aumentar la edad(12), se hipotetiza que el protocolo propuesto permite registrar las diferencias de fluorescencia dental de dientes anteriores en el espacio de color CIELab en distintos grupos etarios.

\section{MATERIALES Y MÉTODOS}

\section{Determinación población de estudio}

Este estudio fue aprobado por el Comité Ético Científico de la Facultad de Odontología de la Universidad de Chile (protocolo de estudio $N^{0}$ 2015/08). Se incluyeron 35 pacientes de entre 21 y 70 años que aceptaron participar en el estudio luego de leer y firmar el formulario de consentimiento informado.

El cálculo de la muestra se hizo utilizando el software G*Power 3.1.2.14 basado en las diferencias en valores $L, a^{*} y b^{*}$ reportado en el estudio "Dental fluorescence: Potential forensic use"(12), considerando un poder estadístico de 0,95 y un valor $\alpha=0,05$. Se determinó una muestra de 70 dientes, 14 por grupo etario:

Grupo 1: 14 dientes de pacientes entre 21 y 30 años

Grupo 2: 14 dientes de pacientes entre 31 y 40 años

Grupo 3: 14 dientes de pacientes entre 41 y 50 años

Grupo 4: 14 dientes de pacientes entre 51 y 60 años

Grupo 5: 14 dientes de pacientes entre 61 y 70 años

Se incluyeron pacientes atendidos durante el segundo semestre del año 2017, en la Facultad de Odontología de la Universidad de Chile, en las clínicas de adultos de cuarto y quinto año, hasta alcanzar el número de pacientes determinados para el estudio, los que debían tener sus 4 incisivos superiores permanentes sin restauraciones ni rehabilitación protésica y, además, que no hubieran recibido tratamiento de blanqueamiento dental previo. Se excluyeron los dientes con índice BEWE superior a 1 en su cara vestibular, o con índice igual o superior a 1 en clasificación para dientes teñidos por tetraciclina de Jordan y Boksman o en el índice de fluorosis de Dean. También se excluyeron pacientes en tratamiento de ortodoncia, con sus incisivos centrales superiores marcadamente rotados o en mala posición y aquellos en que los incisivos centrales superiores tuvieran fracturas y/o lesiones cervicales no cariosas.

Se fotografiaron los incisivos centrales superiores de cada paciente, en un cuarto oscuro privado de cualquier fuente de iluminación natural o artificial externa, lo cual fue confirmado con tres mediciones a distinta y a la misma altura de los incisivos usando un fotómetro Sekonic Litemaster Pro L-478DR-U (Sekonic Corporation, Tokio, Japón) sensible a iluminación en $L x$ desde 0 a 50.000. La lectura en todos los casos fue "Under", significando valores bajo 0 . El paciente usó un separador de mejillas para adulto (Spandex, Promisee Dental Co.Ltda, Huanghua, China) y los dientes fueron iluminados con una linterna zoom LED Convoy (Intsun SK66 S2+, Convoy, China) con una longitud de onda de 365nm dispuesta en un ángulo de $45^{\circ}$ respecto a la superficie dentaria, iluminando sólo el área a fotografiar por el tiempo necesario para hacer la fotografia. Además, a la linterna se le montó un filtro U-340 UV Shortpass IR longpass $37 \mathrm{~mm}$, (Hoya Corporation, CA, USA), el cual permite sólo el paso de luz UV a través de él. Para el procesamiento posterior de las imágenes, se adhirió en el diente 2.2 un disco negro de una tarjeta para balance fotográfico (OPT-DGC-Medium, Opteka, USA). Durante todo el procedimiento, tanto pacientes como operador utilizaron lentes de protección con filtro UV (Spy UV, Redline, China)
Para el registro fotográfico se usó una cámara digital Canon EOS Rebel SL1 (Canon Inc. Japón) con lente macro de 100 mm (Canon Inc. Japón) y un filtro para luz UV e infrarrojo (Fotga Optical Glass, Guangdong, China), configurada con una velocidad de obturación de 1/160, f 2.8, ISO 800, balance de blancos $7000^{\circ} \mathrm{K}$, medición puntual, formato JPEG large y enfoque manual, a $40 \mathrm{~cm}$. Esta estandarización de la cámara se seleccionó luego de probar diferentes configuraciones que permitieran reproducir fielmente lo visto en boca bajo las condiciones de iluminación del estudio, lo cual fue juzgado por dos examinadores calibrados en comparación de color dental (Cohen Kappa 0,82).

Una vez obtenidas las fotos, se procedió a verificar que todo objeto no fluorescente expresara las coordenadas 0,0,0 en el espacio de color RGB, para ello se abrió cada imagen en el programa de libre disposición Image $\mathrm{J}$ $1.51 \mathrm{p}$ (Wayne Rasband, National Institutes of Health, USA), se seleccionó la herramienta color picker y se posicionó sobre el disco negro ubicado sobre el diente 2.2. Una vez confirmado este valor en $0,0,0$, las imágenes fueron analizadas en el espacio de color CIELab, para ello se seleccionó secuencialmente las pestañas image/type/Lab stack lo que generó tres imágenes cada una correspondiente a cada dimensión. El área por medir de cada diente se seleccionó usando la herramienta Polygon selections para lo cual basta seleccionar esta área en la primera imagen, luego de lo cual se procedió a medir los valores Lab usando las pestañas Plugins/ stacks/measure stacks. El programa entregó los valores $L, a^{*}$ y $b^{*}$ de la selección, los que fueron registrados en una hoja de cálculo (Microsoft Excel 2006) para su posterior análisis estadístico. Para determinar la magnitud de la fluorescencia visible inducida por luz UV se calculó la distancia absoluta desde el punto $L=0, a^{*}=0, b^{*}=0$ (inexistencia de color) a través de la fórmula de diferencia de color CIEDE2000(15), utilizando el complemento para Excel "ColorTools" (http://rgbcmyk.com.ar/es/xla/) y se obtuvo el promedio para cada grupo etario.

Los resultados obtenidos fueron analizados mediante el software Statistica, version 13 (TIBCO Software Inc, CA, USA). Se utilizaron las pruebas estadísticas de Shapiro-Wilk (para evaluar la distribución de los datos) y de ANOVA y post-hoc de Tukey HSD para comparar los datos de las coordenadas $L^{*}, a^{*}$ y $b^{*}$ y de magnitud de fluorescencia entre los grupos. Se consideraron estadísticamente significativos valores $p<0,05$.

\section{RESULTADOS}

Todos los grupos mostraron una distribución normal de sus datos, para cada variable evaluada (Shapiro-Wilk $p>0,05$ )

Los valores de las coordenadas $L, a^{*}$ y b* obtenidos para cada grupo etario se muestran en la tabla 1 y las figuras 1,2 y 3.

Tabla 1: Valores de las coordenadas $L, a^{*}$ y $b^{*}$ de cada grupo evaluado, expresado como promedio ( \pm desviación estándar) y comparación entre grupos mediante la prueba ANOVA.

\begin{tabular}{l|c|c|c|c}
\hline & $\mathbf{n}$ & $\mathbf{L}$ & $\mathbf{a}^{*}$ & $\mathbf{b}^{*}$ \\
\hline Grupo 1 & 14 & $\begin{array}{c}52,61 \\
( \pm 10,34)\end{array}$ & $\begin{array}{c}-22,22 \\
( \pm 2,64)\end{array}$ & $\begin{array}{c}-14,17 \\
( \pm 4,07)\end{array}$ \\
\hline Grupo 2 & 14 & $\begin{array}{c}43,35 \\
( \pm 13,03)\end{array}$ & $\begin{array}{c}-14,67 \\
( \pm 4,01)\end{array}$ & $\begin{array}{c}-19,72 \\
( \pm 8,09)\end{array}$ \\
\hline Grupo 3 & 14 & $\begin{array}{c}42,14 \\
( \pm 9,91)\end{array}$ & $\begin{array}{c}-16,66 \\
( \pm 3,39)\end{array}$ & $\begin{array}{c}-14,84 \\
( \pm 6,82)\end{array}$ \\
\hline Grupo 4 & 14 & $\begin{array}{c}42,95 \\
( \pm 13,91)\end{array}$ & $\begin{array}{c}-17,64 \\
( \pm 5,54)\end{array}$ & $\begin{array}{c}-13,79 \\
( \pm 4,54)\end{array}$ \\
\hline Grupo 5 & 14 & $\begin{array}{c}33,15 \\
( \pm 6,15)\end{array}$ & $\begin{array}{c}-15,44 \\
( \pm 2,25)\end{array}$ & $\begin{array}{c}-10,2 \\
( \pm 3,61)\end{array}$ \\
\hline p (ANOVA) & & $\mathbf{0 , 0 0 1}$ & $\mathbf{0 , 0 0 0}$ & $\mathbf{0 , 0 0 1}$ \\
\hline
\end{tabular}

Respecto a la coordenada $\mathrm{L}$, se observó que existe diferencia estadísticamente significativa de los valores entre los grupos $(p<0,005)$. El grupo 1 mostró el mayor valor, estadísticamente diferente al grupo 5 (menor valor) $(p<0,005)$. No hubo diferencia entre los demás grupos.

La coordenada $a^{*}$ mostró que los 5 grupos se ubican en el sector negativo de este eje (espacio verde), con diferencias estadísticamente significativas en sus valores $(p<0,005)$. El grupo 1 mostró el mayor valor en este eje, estadísticamente diferente de los otros grupos $(p<0,005)$. No se encontraron diferencias entre los otros grupos.

En el eje $b^{*}$, se observó a todos los grupos ubicados en el sector negativo (espacio azul), con diferencias entre ellos, con el grupo 2 mostrando los mayores valores, estadísticamente diferentes a los del grupo 5 que mostró los menores valores dentro del eje $(p<0,005)$. No hubo diferencias entre los 


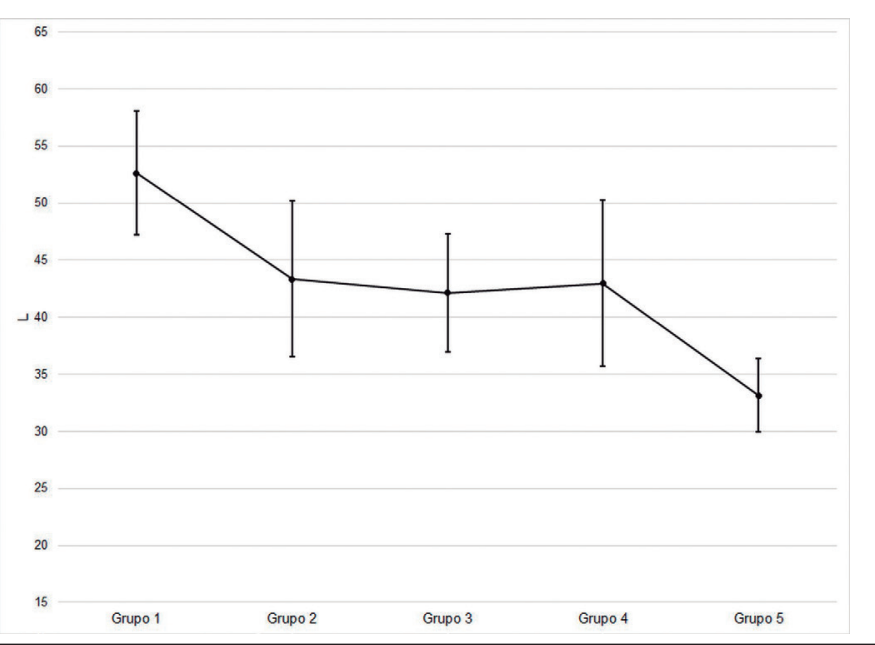

Figura 1. Promedio de valor $L$ en cada grupo. Las barras verticales denotan intervalos de confianza de 0,95.

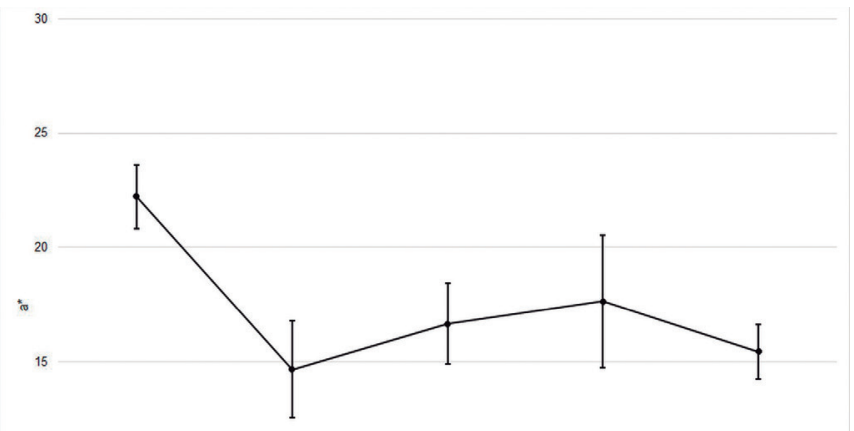

Grupo

Grupo 2

Grupo 3

Grupo 4

Grupo 5

Figura 2. Promedio de valor $a^{*}$ en cada grupo, expresado como magnitud escalar. Las barras verticales denotan intervalos de confianza de 0,95.

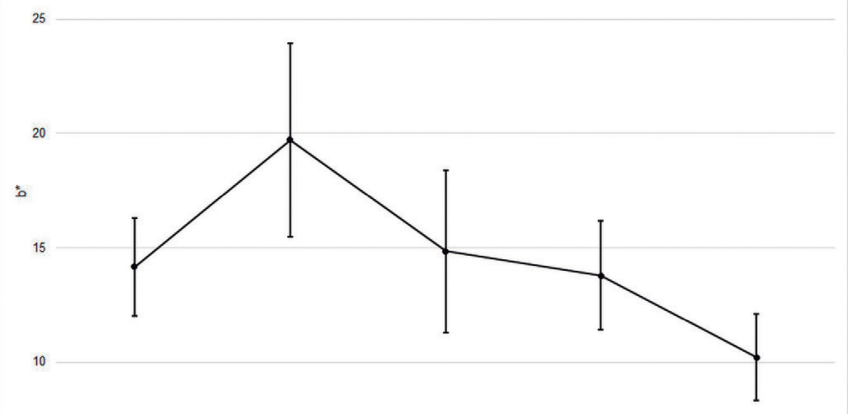

Grupo $1 \quad$ Grupo $2 \quad$ Grupo $3 \quad$ Grupo $4 \quad$ Grupo 5

Figura 3. Promedio de valor $b^{*}$ en cada grupo, expresado como magnitud escalar. Las barras verticales denotan intervalos de confianza de 0,95.

demás grupos.

Al evaluar la intensidad de la fluorescencia emitida por luz UV se observa que el grupo 1 tuvo la mayor intensidad, representado por una mayor distancia respecto al punto $L=0, a^{*}=0, b^{*}=0$, mientras el grupo 5 la menor intensidad (tabla 2). Solo hubo diferencia estadísticamente significativa en los valores de intensidad de fluorescencia al comparar los grupos 1 con el $5(p<0,005)$. No hubo diferencias entre los otros grupos.

La tabla 3 muestra el valor de CIEDE2000 ( $\triangle E 00)$ entre los distintos grupos. El menor valor se encontró entre los grupos 3 y 4 y la mayor diferencia entre los grupos 1 y el 5 .

Con los datos obtenidos y utilizando el software $G^{\star} P o w e r ~ 3.1 .2^{(14)}$, se calculó el poder estadístico de las pruebas utilizadas. Para todas las variables analizadas, el poder estadístico fue de 1,000.

Tabla 2: Intensidad de fluorescencia emitida por luz UV de cada grupo evaluado, representado como diferencia de color CIEDE2000 desde el punto $L=0, a^{*}=0$ y $b^{*}=0$, expresado como promedio ( \pm desviación estándar).

\begin{tabular}{l|c|c}
\hline & $\mathbf{n}$ & DELTA E 2000 \\
\hline Grupo 1 & 14 & $44,25( \pm 9,30)$ \\
\hline Grupo 2 & 14 & $36,10( \pm 10,85)$ \\
\hline Grupo 3 & 14 & $34,77( \pm 8,19)$ \\
\hline Grupo 4 & 14 & $35,89( \pm 11,26)$ \\
\hline Grupo 5 & 14 & $27,52( \pm 4,63)$ \\
\hline p (ANOVA) & & $\mathbf{0 , 0 0 0}$ \\
\hline
\end{tabular}

Tabla 3: Diferencia de color CIEDE2000 entre los distintos grupos

\begin{tabular}{l|c|c|c|c}
\hline & Grupo 1 & Grupo 2 & Grupo 3 & Grupo 4 \\
\hline Grupo 2 & 11,12 & & & \\
\hline Grupo 3 & 10,86 & 3,57 & & \\
\hline Grupo 4 & 9,89 & 4,41 & 1,29 & \\
\hline Grupo 5 & 18,34 & 10,56 & 8,15 & 8,71 \\
\hline
\end{tabular}

\section{DISCUSIÓN}

El protocolo aplicado permitió generar y cuantificar FVIUV en 70 incisivos centrales de sujetos de distintos grupos etarios. Se estableció que todo objeto no fluorescente debía expresar coordenadas CIELab $0,0,0$. Por esto, cualquier valor diferente fue considerado FVIUV.

Si bien el ojo humano no es capaz de discriminar las tres dimensiones de color por separado(16), y comprendiendo que los tres valores resumen un único color (por ejemplo L*a*b* 93,55/-21,76/-14,81 a "verdeazuladoclaro" o cian), el análisis se hace examinando separadamente las 3 coordenadas $L^{*} a^{*} b^{*}$.

Al analizar los resultados en la coordenada $L$, ésta resultó significativamente mayor en los dientes del grupo etario más joven al compararlo con el de mayor edad. Los dientes de los grupos etarios intermedios manifestaron una disminución de ésta, pero sin valores estadísticamente significativos. Estos resultados concuerdan con los obtenidos por Da Silva et al ${ }^{(12)}$, quienes encontraron que el valor máximo de luminosidad estuvo en el grupo etario de 21-30 años y después decaía linealmente al aumentar la edad. Por otro lado, esta coordenada fue usada como única dimensión para cuantificar fluorescencia en el estudio de Conceição et $a^{\left({ }^{(13)}\right.}$, determinación controversial, ya que si bien es cierto ha sido probado matemáticamente que la luminosidad establece una mayor influencia en la diferencia total de color, la influencia de saturación y tono en el color final también es relevante ${ }^{(16)}$

Con respecto a la coordenada $a^{*}$, que varía desde el tono a+ (rojo) hasta el tono a- (verde), se detectó variaciones relacionadas con la edad. Todos los grupos etarios mostraron FVIUV dentro del color verde con diferencias estadísticamente significativas en sus valores de saturación, siendo el grupo etario más joven el con más alta saturación. Por otro lado, La coordenada $b^{*}$, que va desde el tono b+ (amarillo) hasta el tono b- (azul), también presentó variaciones en relación con la edad. Se observó que en todos los grupos la coordenada se mantuvo en el rango del color azul, siendo el grupo de mayor edad el con los valores más bajos de saturación. Estos resultados concuerdan con estudios espectrofotométricos de fluorescencia dentaria en que la longitud de onda predominante se encuentra en los rangos de $450 \mathrm{~nm}$ a $528 \mathrm{~nm}^{(1,6,8)}$, lo cual se encuentra entre azul y verde del espectro de radiación electromagnética ${ }^{(17,18)}$

En el estudio de Da Silva et al se obtuvieron resultados diferentes en lo que se refiere a la coordenada $a^{*}$, ya que en él todos los grupos etarios se mantenían dentro del rango del color rojo ${ }^{(12)}$, lo cual no es congruente con los estudios de mediciones espectrofotométricas ya mencionados ${ }^{(1,6,8)}$.

Para determinar la magnitud de la FVIUV en el presente trabajo se utilizó la fórmula de diferencia de color CIEDE2000 ( $\Delta \mathrm{E} 00)$ debido a que esta fórmula refleja mejor las diferencias de color percibidas por el ojo humano que la fórmula $\operatorname{CIELab}(\Delta \mathrm{E} * \mathrm{ab})^{(19,20)}$. El punto de partida fueron las coordenadas CIELab 0,0,0 que expresan ausencia de color, a partir 
de este punto se fue cuantificando la diferencia que con respecto a él presentaba cada uno de los grupos etarios. El que más se alejó de ese punto fue el grupo de menor edad con un $\Delta \mathrm{E} 0044,25$, por lo tanto, teniendo la mayor magnitud de FVIUV, y el que menos se alejó, menor FVIUV, fue el grupo de mayor edad con un $\triangle \mathrm{E} 00$ de 27,52 .

Para analizar las diferencias de magnitud de color $\Delta \mathrm{E} 00$ entre grupos desde una perspectiva de la percepción humana, se consideró los Umbrales de Percepción y de Tolerancia. El umbral de percepción (50:50\% PT, por sus siglas en inglés) indica que cuando la diferencia de magnitud de $\triangle \mathrm{E} 00$ entre un objeto y otro es superior a 0,8 el $50 \%$ de los observadores son capaces de percibir diferencias de color. Por otra parte, el umbral de aceptación (50:50\% AT, por sus siglas en inglés) establece que el $50 \%$ de los observadores juzga la diferencia de color aceptable solo hasta un umbral de $1,8^{(19)}$. Por lo anterior, podemos aseverar que, las diferencias de color entre los distintos grupos etarios serían percibidas claramente por cualquier observador y con la sola excepción de la diferencia entre los grupos 3 y 4 , éstas serían juzgadas como inaceptables o muy marcadas.

La importancia de los resultados del presente estudio radica en ofrecer una alternativa para incorporar la fluorescencia al momento de diagnosticar el color dentario, permitiendo mediante el uso de una cámara fotográfica digital, el apoyo de filtros, linterna LED UV y un programa de libre disposición obtener sus magnitudes en el espacio de color CIELab. Este protocolo permitiría a través de un proceso de comparación, seleccionar y evaluar materiales restauradores en clínica. Más aún, gracias al presente estudio se verificó que debido a los altos valores de $\Delta \mathrm{E} 00$, la diferencia de FVIUV entre distintos grupos etarios puede ser notada directamente por el observador promedio.

De esta manera, bajo condiciones clínicas, se podría proyectar que mediante la iluminación con LED UV, primero, se debieran hacer evidentes las características de fluorescencia propias de la edad del paciente, segundo, si un material restaurador fuera adecuado en su FVIUV para un determinado grupo etario, sería altamente probable que no lo fuera para un grupo de distinta edad, y tercero, se debería tener presente que los materiales de restauración pueden presentar una alta variabilidad de colores al fluorescer según la marca y el tono del material(21).

Este estudio buscó perfeccionar y simplificar un protocolo anteriormente descrito $^{(12)}$. En primer lugar, para eliminar cualquier posibilidad de interferencia de radiaciones contaminantes en el momento de captación de la imagen, lo primero que se hizo fue filtrar la radiación que emitía la linterna LED UVA de $365 \mathrm{~nm}$, ya que la caracterización habitual de lámparas de luz UVA o lámparas de UVB es imprecisa, debido a que todas ellas emiten UVA, UVB e incluso UVC, luz visible y radiación infrarroja debido a limitaciones en protocolos de calibración y estandarización en su fabricación ${ }^{(22,23)}$. El Filtro usado sobre la linterna permitió el paso de un estrecho margen de radiación UV en el rango de $260 \mathrm{~nm}$ a $390 \mathrm{~nm}$, bloqueando el paso de luz visible, pero permitiendo el paso de algo de radiación infrarroja en el rango de $680 \mathrm{~nm}$ a $780 \mathrm{~nm}^{(24)}$. Para solucionar esto, en el lente de la cámara fotográfica se puso un filtro que bloqueó el paso a través de él de cualquier otra radiación del espectro que no fuera luz visible.

El uso de filtros evita captación de luz que no corresponda a la FVIUV y que en estudio de Da Silva(12) podría haber incidido en que en la coordenada $a^{*}$ se obtuvieran valores rojos.

En lo referente a simplificación, en el análisis de las imágenes en vez de software comercial, se incorporó el uso un software de libre disposición y de uso amigable.

A modo de limitación, se debe hacer presente que las imágenes obtenidas mediante fotografía digital pueden ser afectadas en su confiabilidad y precisión al ser influenciadas por las condiciones ambientales, la fuente lumínica y también especificaciones técnicas de la cámara digital y su configuración ${ }^{(25)}$. Por esto, hay que considerar que los resultados aquí obtenidos son válidos bajo la estandarización implementada en el presente estudio. En ese sentido, el valor que tiene usar cámara fotográfica digital es permitir registros estandarizados apuntando a la cuantificación de fenómenos subjetivos en la practica clínica diaria.

El presente estudio ha servido para darse cuenta que al comparar distintos grupos etarios, las diferencias de color dentario generadas por la exposición a radiación UV pueden ser evidentes al observador, y que usando un protocolo simple es posible lograr su cuantificación con resultados congruentes a los logrados usando otras tecnologías y protocolos ${ }^{(12,13)}$.

Lo anterior es importante en los tiempos presentes en que la población suele pasar un alto porcentaje de tiempo bajo iluminación artificial, las cuales tienen importantes componentes de radiación UV(10) que puede hacer evidente las diferencias de color entre una restauración y el diente debido a su diferente FVIUV.

De igual modo, el protocolo aquí descrito podría ser de útil aplicación en la Odontología Forense tanto por su simplicidad y rapidez de ejecución, como a que su uso permite evidenciar variaciones de fluorescencia dentaria inducida por luz UV según edad del paciente.

En conclusión, El protocolo aplicado en presente estudio permitió la cuantificación de la FVIUV dentaria en distintos grupos etarios, detectando FVIUV en el espectro del color verde azulado y variaciones de saturación y luminosidad con tendencia a disminuir con la edad.

\section{CONFLICTO DE INTERÉS}

Los autores declaran no tener conflictos de Interés.

\section{Bibliografía}

1. Hall JB, Hefferren JJ, Olsen NH. Study of fluorescent characteristics of extracted human teeth by use of a clinical fluorometer. J Dent Res. 1970;49(6):1431-6.

2. Park M-Y, Lee Y-K, Lim B-S. Influence of fluorescent whitening agent on the fluorescent emission of resin composites. Dent Mater. 2007;23(6):731-5.

3. Espinosa Ipinza F, Rivas Poblete V. Fluorescencia visible inducida por radiación UV: sus usos en conservación y diagnóstico de colecciones: una revisión crítica. Conserva. 2011;(16):27-38

4. Yu B, Lee YK. Differences in color, translucency and fluorescence between flowable and universal resin composites. J Dent. 2008;36(10):840-6.

5. Takahashi MK, Vieira S, Rached RN, Almeida JB, Aguiar M, Souza EM Fluorescence intensity of resin composites and dental tissues before and after accelerated aging: a comparative study. Oper Dent. 2008;33(2):189-95.

6 . Lee Y-K. Fluorescence properties of human teeth and dental calculus for clinical applications. J Biomed Opt. 2015;20(4):1-8.

7. Hein S, Ten Bosch JJ. The effect of ultraviolet induced fluorescence on visually perceived tooth color under normal light conditions. Dent Mater. 2018;34(5):819-23 8. Dickson G, Forziati AF, Lawson ME, Schoonover IC. Fluorescence of teeth; a means of investigating their structure. J Am Dent Assoc. 1952;45(6):661-7.

9. Volpato CAM, Pereira MRC, Silva FS. Fluorescence of natural teeth and restorative materials, methods for analysis and quantification: A literature review. $J$ Esthet Restor Dent. 2018 Sep;30(5):397-407.

10. Cebula TA, Henrikson EN, Hartman PE, Biggley WH. Reversion profiles of cool white fluorescent light compared with far ultraviolet light: homologies and differences. Photochem Photobiol. 1995;61(4):353-9.

11. Hermanson AS, Bush MA, Miller RG, Bush PJ. Ultraviolet illumination as an adjunctive aid in dental inspection. J Forensic Sci. 2008;53(2):408-11.

12. Da Silva RD, da Silva MAD, de Oliveira OB, Melo ACM, de Oliveira RN. Dental fluorescence: Potential forensic use. Forensic Sci Int. 2013;231:167-71.

13. Conceição LD, Masotti AS, Forgie AH, Leite FRM. New fluorescence and reflectance analyses to aid dental material detection in human identification Forensic Sci Int. 2019;305:110032.
14. Faul F, Erdfelder E, Lang A-G, Buchner A. G* Power 3 : A flexible statistical power analysis program for the social, behavioral, and biomedical sciences. Behav Res Methods. 2007;39(2):175-91.

15. Sharma G, Wu W, Dalal EN. The CIEDE2000 color-difference formula: Implementation notes, supplementary test data, and mathematical observations. Color Res Appl. 2005;30(1):21-30

16. Paravina RD, Powers JM, Fay RM. Dental color standards: shade tab arrangement. J Esthet Restor Dent. 2001;13(4):254-63.

17. Bohren CF, Clothiaux EE. Fundamentals of atmospheric radiation: an introduction with 400 problems. Wiley; 2006.

18. ISO 21348 Definitions of Solar Irradiance Spectral Categories. [cited $2020 \mathrm{Ju}$ 21]. Available from: http://www.spacewx.com/pdf/SET_21348_2004.pdf

19. Paravina RD, Ghinea R, Herrera LJ, Bona AD, Igiel C, Linninger M, et al. Color difference thresholds in dentistry. J Esthet Restor Dent. 2015;27:S1-9.

20. Gómez-Polo C, Muñoz MP, Lorenzo Luengo MC, Vicente P, Galindo P, Martín Casado AM. Comparison of the CIELab and CIEDE2000 color difference formulas. J Prosthet Dent. 2016 Jan;115(1):65-70.

21. Lim Y-K, Lee Y-K. Fluorescent emission of varied shades of resin composites. Dent Mater. 2007 Oct;23(10):1262-8

22. Diffey BL. Sources and measurement of ultraviolet radiation. Methods 2002;28:4-13

23. Eppeldauer GP. Standardization of broadband uv measurements for $365 \mathrm{~nm}$ LED sources. J Res Natl Inst Stand Technol. 2012;117:96-103.

24. Hoya Optics [Internet]. [cited 2020 Jul 21]. Available from: https://hoyaoptics. com/wp-content/uploads/2019/10/U340.pdf

25. Knösel M, Attin R, Jung K, Brunner E, Kubein-Meesenburg D, Attin T. Digital image color analysis compared to direct dental CIE colorimeter assessment under different ambient conditions. Am J Dent. 2009;22(2):67-72. 